\title{
Stimulators and simulation models of the brain from viewpoints of diabetes complications (GH-Method: math-physical medicine)
}

Gerald C. Hsu

eclaireMD Foundation, USA

\author{
Correspondence author \\ Gerald C. Hsu \\ eclaireMD Foundation \\ USA
}

Submitted : 17 June 2020 ; Published : 2 July 2020

\section{Introduction}

This paper describes stimulators and simulation models of the human brain from viewpoints of diabetes complications, including the brain, heart, kidney, and eyes.

\section{Methods and Background}

The author spent 24,000 hours for the past 9-years from 2010 to 2019 , to conduct research on diabetes disease and its various complications by using his GH-Method: mathphysical medicine (MPM) methodology. As he delved deeper into diabetes, he realized the significant role the brain played in this disease and its complications. Actually, his application of various research tools, including physics, mathematics, engineering modeling, computer science, and artificial intelligence (AI) all use the analysis part of the brain in relation to another section of it within the body's biological operations. Now, he is trying to interpret his research findings and conclusions via a stimulator identification and simulation model definition of the brain.

Initially, there are some "baseline conditions" to be determined before analyzing further regarding diabetes complications, including heart attack (cardiovascular disease), stroke, kidney (renal disease), and eyes (retinal disease). These "static" baseline factors of chronic diseases include age, which is most important, race, gender, family history, personal bad habits such as drinking, smoking, and substance abuse, along with good habits such as healthy diet and sufficient exercise. Although it is debatable, the author would like to include two extra "semi-static" conditions, weight and waistline, into this baseline category since these they are not easily changed over a short period of time. These static baseline factors are either registered inside the DNA or imprinted (i.e. stored) inside the brain to be served as the "Master Database".

\section{Stimulators}

In terms of stimulators, there are two categories. First category includes heart attack and stroke which are caused by artery damage from three primary stimulators: aging, weight, and glucose. The two secondary stimulators involve blood pressure and lipids. The second category includes renal and retinal complications which are caused by micro-vessel damage from the same three primary stimulators mentioned earlier and one secondary stimulator, blood pressure. Lipids are excluded here due to the micro-vessel's minuscule size which they cannot easily pass through. Blood pressure always exist in the blood flow regardless of the blood vessel's size.

\section{Simulation Models}

Since aging and weight cannot be modified or changed easily, the only remaining "dynamic" (change with time) factor is glucose. In the human body, glucose acts like a "double-edged sword". It serves as the source of energy or metabolism which circulates throughout the internal organs within the blood flow. However, excessive energy generated by high glucoses over a long period of time also damages the internal organs (see references).

The author applied his knowledge of physics and engineering to build up several simulation models.

He has built two artery simulation models: blood vessel using structure dynamics and blood flow using fluid dynamics.

Let us discuss the simulation model for the first situation: artery damage. Due to aging, similar to a water pipe, the artery vessel's structure would be weakened over time. Along with this effect, high glucose impact on blood vessels is like having acid placed on the water pipe. Therefore, the combination of aging and glucose would affect the blood vessel's structural integrity. Moreover, the immune cells in the artery vessel would view hyperglycemia as an "intruder" and fight against it, 
causing inflammation and creating rough vessel surface. This rough surface friction would cause lipids to be deposited at that location and eventually build up a blood flow blockage, which is like having butter flowing through a water pipe. Sometimes, hypertension precedes the high lipids issue, resulting with a vessel rupture that is similar to a broken water pipe under high water pressure. Depending on the actual location of the blood flow blockage or blood vessel rupture, a patient would consequently suffer a heart attack or a stroke.

For the second situation, the author developed a blood microvessel simulation models by using only structure dynamics. The micro-vessel's primary stimulators, aging and glucose, are the same as the artery case. However, due to the small size of the vessel, lipids cannot pass through easily and therefore, has no significant impact on the micro-vessel's structural integrity and flow. With the second stimulator, blood pressure, combined with the primary stimulators, aging and glucose, it will create many tiny holes on the micro-vessel's surface. In the kidneys, proteins would leak out of these holes into urine and leave wastes inside this particular organ which increases the albumin-to-creatinine ratio (ACR), a marker for renal damage.

\section{Results}

Based on these identified brain stimulators and developed brain simulation models involving diabetes complications, the author derived a set of AI-based risk probability equations to estimate varying probability percentages for the patient in developing different severity stages of diabetes complications, including CVD, stroke, and renal complications.

He used these equations and his collected 1.5 million personal data set for the past 9-years, from 2010 to 2019, which includes both "static" baseline and "dynamic" disease stimulators, including glucose, blood pressure, and lipids to calculate his own risk probabilities on an annual basis.

\section{The key results are shown below.}

\section{0-2012 (initial years):}

1. CVD and stroke: $69 \%-84 \%$ - he suffered five cardiac episodes from 2000 to 2009.

2. Renal: $80 \%$ - doctors urged him to start dialysis in 2010 .

\section{7 (under control year):}

1. CVD and stroke: $25 \%$ to $28 \%$

2. Renal: $31 \%$

\section{Conclusions}

All of these data-processing and data-analyzing tasks are performed naturally by the brain. The author is merely discovering certain facts and proving some of his hypotheses through relevant data collection, physical phenomena observation, statistical calculation, appropriate mathematical equations derivation, and vital output's prediction. It is the brain doing the actual important analysis work and decisionmaking and registering vital bio-signs such as weight, glucose,
BP, lipids, and more. He is simply trying to understand different stimulators, develop corresponding and adequate simulation models, and then discover some hidden physical characteristics, behavior patterns, and relationships which are already managed and produced by the brain. One important final step for the author is to derive suitable mathematical equations in order to calculate and predict various risk probabilities of different complications based on his hypotheses and discoveries.

Both traditional biochemical medicine (BCM) and the author's math-physical medicine (MPM) are two different simulation methods of the human brain, which can be used to understand its various functions, multiple activities, and complicated albeit effective decision-making process. Through distinct and hopefully complimentary research methodologies, cross disciplinary trainings, and the use of different but practical approximation tools, the author anticipates that we will eventually be able to better understand the brain's stimulators and its related simulation models regarding disease complications.

This article is not striving to analyze the brain from the viewpoints of traditional neuroscience or neurology, it is rather emphasizing either BCM or MPM approaches as different yet having the same goals to understand the brain stimulators and related brain simulation models [1-5].

\section{References}

1. Hsu, Gerald C (2018) Using Math-Physical Medicine to Control T2D via Metabolism Monitoring and Glucose Predictions. Journal of Endocrinology and Diabetes 1(1): 1-6.

2. Hsu, Gerald C (2018) Using Signal Processing Techniques to Predict PPG for T2D. International Journal of Diabetes \& Metabolic Disorders 3(2): 1-3.

3. Hsu, Gerald C (2018) Using Math-Physical Medicine and Artificial Intelligence Technology to Manage Lifestyle and Control Metabolic Conditions of T2D. International Journal of Diabetes \& Its Complications 2(3): 1-7.

4. Hsu, Gerald C (2018) Using Math-Physical Medicine to Analyze Metabolism and Improve Health Conditions. Video presented at the meeting of the 3rd International Conference on Endocrinology and Metabolic Syndrome 2018, Amsterdam, Netherlands.

5. Hsu, Gerald C (2018) Using Math-Physical Medicine to Study the Risk Probability of having a Heart Attack or Stroke Based on Three Approaches, Medical Conditions, Lifestyle Management Details, and Metabolic Index. EC Cardiology 5(12): 1-9.

Copyright: (C2020 Gerald C. Hsu. This is an open-access article distributed under the terms of the Creative Commons Attribution License, which permits unrestricted use, distribution, and reproduction in any medium, provided the original author and source are credited. 